\title{
Aplicativo móvel para diagnóstico das desordens temporomandibulares articulares: desenvolvimento do protótipo
}

Mobile application for diagnosis of articular temporomandibular disorders: prototype development Aplicación móvil para diagnóstico de los desordes temporomandibulares articulares: desarrollo del protótipo

Maritza Monçalves Viana CORTELLETTI ${ }^{1}$

Gabriela Moraes MACHADO ${ }^{1}$

Rafaela Hartmann KASPER ${ }^{1}$ Raul Antônio CRUZ²

Caren Serra BAVARESCO${ }^{3}$

\author{
${ }^{1}$ Acadêmica do curso de Odontologia - ULBRA Univ. Luterana do Brasil, 92425-020 Canoas - RS, Brasil \\ ${ }^{2}$ Especialista em Disfunção temporomandibular e Dor Orofacial. Professor do Curso de Graduação em Odontologia \\ ULBRA Univ Luterana do Brasil, 92425-020 Canoas - RS, Brasil \\ ${ }^{3}$ Cirurgiã-Dentista, Doutora em Ciências Biológicas: Bioquímica - UFRGS. Professora do Curso de Graduação em Odontologia \\ ULBRA Univ. Luterana do Brasil, 92425-020 Canoas - RS, Brasil
}

\section{Resumo}

Introdução: Em função da importância do diagnóstico precoce e correto das inúmeras patologias que podem afetar a Articulação Temporomandibular e das dificuldades em diagnosticar apresentadas pelos profissionais da Odontologia, mostra-se necessário propor mecanismos que sejam auxiliadores nesse procedimento. Objetivo: Desenvolver um aplicativo móvel com ênfase no diagnóstico das Disfunções Temporomandibulares Articulares, tendo como público alvo os profissionais de saúde bucal e graduandos em Odontologia. Material e Método: Foi desenvolvido um sistema caracterizado pelo preenchimento de um questionário contendo as principais características das DTMs segundo a Academia Americana de Dor. Em relação à plataforma, optou-se por utilizar o sistema Android, e, quanto ao gerenciador de dados, foi utilizado o MySQL. O questionário inicia-se com o Índice de Fonseca a fim de identificar se o paciente é portador de DTM. Caso o paciente seja portador de DTM, o questionário seguirá para questões relacionadas ao diagnóstico de DTMs articular. Neste primeiro momento do protótipo, foram introduzidas dez perguntas no questionário sobre o deslocamento do disco com redução. Resultados: O aplicativo móvel para diagnóstico das desordens temporomandibulares articulares apresentou funcionalidade capaz de disponibilizar um escore de severidade de DTMs articular do paciente e diagnóstico de deslocamento do disco com redução. Conclusão: O desenvolvimento de aplicativos móveis direcionados ao manejo da DTM é relevante, já que a prevalência desta patologia é crescente em termos populacionais, sendo necessário que o cirurgião-dentista esteja capacitado para realizar o diagnóstico correto e propor estratégias de tratamento.

Descritores: Aplicativos Móveis; Síndrome da Disfunção da Articulação Temporomandibular; Diagnóstico.

\section{Abstract}

Introduction: Due to the importance of early and correct diagnosis of the countless pathologies that can affect the Temporomandibular Joint and the difficulties in diagnosing presented by dentistry professionals, it is necessary to propose mechanisms that help this procedure. Objective: To develop a mobile application with an emphasis on the diagnosis of Temporomandibular Disorders, with a target audience of oral health professionals and undergraduate dentists. Material and Method: A system was developed characterized by completing a questionnaire containing the main characteristics of TMDs according to the American Academy of Pain. In relation to the platform, it was decided to use the Android system, and for the data manager, MySQL was used. The questionnaire starts with the Fonseca Index in order to identify whether the patient has TMD. If the patient has TMD, the questionnaire will proceed to questions related to the diagnosis of joint TMDs. In this first moment of the prototype, ten questions were introduced in the questionnaire about the displacement of the disc with reduction. Results: The mobile application for the diagnosis of temporomandibular joint disorders presented functionality capable of providing a severity score for the patient's joint TMDs and a diagnosis of disc displacement with reduction. Conclusion: The development of mobile applications directed to the management of TMD is relevant, since the prevalence of this pathology is increasing in population terms, and it is necessary that the dentist is trained to perform the correct diagnosis and propose treatment strategies.

Descriptors: Mobile Applications; Temporomandibular Joint Dysfunction Syndrome; Diagnosis.

\section{Resumen}

Introducción: debido a la importancia del diagnóstico precoz y correcto de las innumerables patologías que pueden afectar la articulación temporomandibular y las dificultades de diagnóstico que presentan los profesionales de la odontología, es necesario proponer mecanismos que ayuden a este procedimiento. Objetivo: desarrollar una aplicación móvil con énfasis en el diagnóstico de los trastornos temporomandibulares, con un público objetivo de profesionales de la salud bucal y dentistas universitarios. Material y método: se desarrolló un sistema que se caracteriza por completar un cuestionario que contiene las características principales de los TMD según la Academia Estadounidense del Dolor. En relación con la plataforma, se decidió utilizar el sistema Android, y para el administrador de datos, se utilizó MySQL. El cuestionario comienza con el Índice Fonseca para identificar si el paciente tiene TMD. Si el paciente tiene TMD, el cuestionario procederá a las preguntas relacionadas con el diagnóstico de TMD conjuntas. En este primer momento del prototipo, se introdujeron diez preguntas en el cuestionario sobre el desplazamiento del disco con reducción. Resultados: la aplicación móvil para el diagnóstico de trastornos de la articulación temporomandibular presentó una funcionalidad capaz de proporcionar una puntuación de gravedad para los TMD articulares del paciente y un diagnóstico de desplazamiento del disco con reducción. Conclusión: El desarrollo de aplicaciones móviles dirigidas al manejo de TMD es relevante, ya que la prevalencia de esta patología está aumentando en términos de población, y es necesario que el dentista esté capacitado para realizar el diagnóstico correcto y proponer estrategias de tratamiento.

Descriptores: Aplicaciones Móviles; Síndrome de la Disfunción de Articulación Temporomandibular; Diagnóstico.

INTRODUÇÃO

As disfunções temporomandibulares (DTM) são desordens músculo-esqueléticas envolvendo a articulação temporomandibular (ATM), os músculos da mastigação e estruturas anexas, resultando em uma perturbação no equilíbrio funcional e dinâmico da $\mathrm{ATM}^{1}$. Esta possui movimento de dobradiça em um plano, característica de uma articulação ginglemoidal, 
ao mesmo tempo em que proporciona movimentos de deslize, o que a classifica como articulação artroidal, associada à presença do disco articular que permite movimentos complexos da articulação ${ }^{2}$.

A DTM apresenta etiologia multifatorial e pode estar associada a fatores dentários e/ou faciais, que se relacionam com o aparelho estomatognático ${ }^{3}$, necessitando, assim, de uma abordagem interdisciplinar para seu tratamento. As dores na região da ATM, cefaleia, dores nos músculos mastigatórios, limitação funcional e estalidos destacam-se como sinais e sintomas. A exacerbação desse quadro clínico acaba por limitar ou mesmo incapacitar o indivíduo em suas atividades cotidianas ${ }^{4}$.

Para o estabelecimento de um correto plano de tratamento, faz-se necessária a avaliação da história do paciente, da realização de exame clínico acurado e, muitas vezes, da presença de exames imaginológicos ${ }^{5}$. Dentre as possibilidades de diagnóstico relacionadas às DTMs, é imprescindível a distinção entre mialgias e artralgias. As mialgias, de acordo com Okeson ${ }^{6}$, é a dor relacionada aos tecidos musculares, podendo variar de uma ligeira sensibilidade até um desconforto extremo, tendo como sintomas associados à sensação de fadiga e tensão muscular. Por outro lado, as artralgias caracterizam-se pela dor em qualquer estrutura articular, originando-se de nociceptores localizados nos tecidos moles ao redor da articulação ${ }^{6}$.

No contexto das artralgias, destacam-se os deslocamentos de disco com ou sem redução, as doenças inflamatórias e as patologias degenerativas. O deslocamento do disco com redução é observado quando, em algum momento dos movimentos mandibulares, o côndilo e o disco articular não ficam corretamente posicionados, retornando posteriormente a posição normal ${ }^{7}$. Durante 0 exame clínico, deslocamento do disco com redução está associado à presença de estalidos na ATM. Depois que o disco é reduzido, a amplitude do movimento mandibular volta ao normal. O deslocamento do disco sem redução ocorre quando a pessoa é incapaz de retornar à posição normal do complexo disco articularcôndilo mandibular. Já no deslocamento agudo sem redução, a boca pode não atingir sua abertura máxima visto que a posição do disco não permite a translação total do côndilo? ${ }^{7}$.

Nas desordens articulares inflamatórias, destacam-se os grupos de sinovite, capsulite e retrodiscite $^{8}$. As sinovites são caracterizadas pela inflamação dos tecidos sinoviais que cercam as áreas da articulação, sendo a dor intracapsular constante. Além disso, é causada por qualquer condição irritativa dentro da articulação, de forma que se acentua pelo movimento. Por outro lado, a capsulite pode ser evidenciada quando o ligamento capsular se torna inflamado, caracterizando-se por uma dor no polo lateral do côndilo durante a palpação, podendo produzir dor até mesmo com a articulação em posição estática. Com o movimento articular, a dor tende a aumentar. Seu fator causal mais comum é o macrotrauma, seguido do colapso e inflamação ${ }^{8}$. A retrodiscite consiste na inflamação dos tecidos retrodiscais e caracteriza-se, clinicamente, por uma dor pulsátil e/ou em choque na região pré-auricular, que piora com a manipulação mandibular. Sinovite, capsulite e retrodiscite são patologias de difícil diferenciação, sendo seu tratamento idêntico ${ }^{8}$.

Não obstante, a artrite é conceituada como mudanças ósseas destrutivas, representada pela osteoartrite que é caracterizada por um processo destrutivo onde as superfícies ósseas articulares do côndilo e fossa se alteram, bem como é uma condição não inflamatória na qual as superfícies articulares e o osso adjacente deterioram-se, sendo uma resposta do próprio organismo ao aumento da carga na articulação ${ }^{8}$. Conforme a carga persiste, a superfície articular se torna mais macia e o osso subarticular começa a ser reabsorvido. Destaca-se que uma degeneração progressiva resultará em perda da camada cortical subcondral e erosão óssea, podendo ser evidenciada radiograficamente apenas em estágios avançados. Em relação às superfícies, quando aparentam estar erodidas e aplainadas, são consideradas como osteoartrite secundária. Quando a alteração do osso está ativa, é frequentemente dolorosa, agravando-se com o movimento mandibular ${ }^{8}$.

A anquilose, por definição, significa a imobilidade anormal da articulação, sendo diferenciada em fibrosa e óssea de acordo com o tecido que limita sua mobilidade. A anquilose fibrosa é a mais comum, ocorrendo entre o côndilo e o disco ou o disco e a fossa mandibular. A anquilose óssea é mais rara, representando uma desordem crônica e extensa que pode ocorrer entre o côndilo e a fossa articular, sendo que a perda do espaço discal é requisito para que isso se configure. Quando a anquilose está presente, a mandíbula não pode transladar da fossa, resultando numa amplitude muito pequena de movimento ${ }^{2}$.

Contudo, a etiologia da DTM é controversa e multifatorial, sendo os traumas, a ausência dentária e uso de próteses citados 
dentre os fatores desencadeantes, além de hábitos parafuncionais como bruxismo $e$ onicofagia. A intensidade e a frequência das atividades parafuncionais podem ser exacerbadas por estresse, nervosismo e ansiedade $^{3,9}$. Os sintomas dessa disfunção têm sido relatados em até $88 \%$ dos indivíduos, sendo $25 \%$ sob a forma de sintomas graves. Muitos estudos indicam que há predominância do gênero feminino entre os portadores de disfunção, e geralmente a população jovem é a mais acometida ${ }^{4-6}$.

Além disso, determinado estudo verificou o grau de conhecimento dos cirurgiões-dentistas sobre etiologia, incidência e diagnóstico das DTMs. Os resultados obtidos indicaram que a maioria dos profissionais possui conhecimento insuficiente não só em relação à anatomia e à fisiologia da ATM e estruturas anexas, mas também das patologias que acometem a mesma (sinais e sintomas) ${ }^{10}$. Por outro lado, outro estudo analisou que no campo de conhecimento das DTMs, a tradição anedótica (ou seja, estudos sem embasamento científico adequado) sempre esteve presente, e continua a influenciar muitas decisões sobre tratamento, bem como no referido estudo a maior parte dos entrevistados não se sentem seguros com relação ao diagnóstico, decisão terapêutica e avaliação dos resultados do tratamento das DTMs ${ }^{11}$.

Sendo assim, evidencia-se a necessidade de construção de alternativas para capacitar o cirurgião-dentista no diagnóstico das DTMs, sendo as alterações articulares as que suscitam maior número de dúvidas devido à sua complexidade em relação ao diagnóstico. Em um contexto de avanço tecnológico e inovação no âmbito de atendimento na área da saúde, o desenvolvimento de aplicativos móveis tem ganhado cada vez mais espaço.

Em função da importância do diagnóstico precoce e correto das inúmeras patologias que podem afetar a ATM e das dificuldades apresentadas pelos profissionais da Odontologia, este trabalho teve por objetivo desenvolver um aplicativo móvel com ênfase no diagnóstico das DTMs, voltado para os profissionais de saúde bucal e graduandos em Odontologia. Salienta-se que esta plataforma digital não tem como propósito substituir qualquer exame complementar por imagem, mas sim apresentar uma forma complementar de auxílio diagnóstico.

\section{MATERIAL E MÉTODO}

O aplicativo móvel foi abastecido através do preenchimento de um questionário contendo as principais características destas patologias, segundo os critérios da Academia Americana de Dor $^{12}$. Destaca-se que a utilização desse aplicativo não exclui outras formas complementares de diagnóstico.

A plataforma utilizada para a construção do aplicativo foi a do sistema operacional mobile Android (nível API 23) para celulares com a versão 4.0 .2 , presente em $100 \%$ dos celulares ativos, conforme dado fornecido pelo Google ${ }^{13}$.

Em relação ao gerenciador de banco de dados, foi utilizado o MySQL, sendo um dos componentes centrais da maioria das aplicações públicas da internet. Ele é gratuito e uma ferramenta eficiente para aplicações Web. O gerenciador de bancos de dados utiliza a linguagem SQL (Structure Query Language Linguagem de Consulta Estruturada), que é a uma linguagem muito popular para inserir, acessar e gerenciar o conteúdo armazenado em um banco de dados. O MyQSL é usado para fazer a comunicação entre o aplicativo e o banco de dados, ou seja, transporta os dados informados pelo usuário e os grava no banco de dados. Os dados relativos ao aplicativo, por meio da utilização do $M y Q S L$, são enviados a um determinado endereço eletrônico, viabilizando um acesso rápido. Além disso, tais dados são registrados em forma de tabela, contendo o nome do usuário, o e-mail, a profissão, o sexo, a data de nascimento, a cidade de domicílio e o grau de conhecimento sobre DTM ${ }^{14}$.

O layout é preconizado pelos guias de documentação de layout para Android que visam padronizar o layout dos aplicativos ${ }^{15}$. Foram selecionados tons variados de azul para o plano de fundo do aplicativo e letras brancas para destacar o conteúdo dos questionários. Quanto ao layout das questões, optou-se por uma questão do questionário por tela, para que ficasse mais claro e com melhor visualização para o usuário.

Com base na literatura, foi realizado um questionário contendo critérios de diagnósticos para DTM articulares, utilizados como base ao aplicativo móvel. Dessa forma, tal questionário inicia-se pelo Índice Anamnésico de Fonseca a fim de identificar se o paciente é portador de DTM. O paciente que for submetido à anamnese terá um diagnóstico efetivo, porém não é descartado nenhum outro tipo de exame complementar.

$O$ Índice Anamnésico de Fonseca ${ }^{16}$ é composto por 10 perguntas no qual as respostas somam pontos que determinam se 0 paciente não é portador de DTM, se a DTM é leve, moderada ou severa.

O questionário diagnóstico para DTM 
Articular é composto por 9 patologias da ATM, segundo os critérios da Academia Americana de Dor:

a) Deslocamento de Disco com redução: contendo 10 critérios de presença obrigatória e 6 de presença complementar;

b) Deslocamento Agudo de Disco sem redução: contendo 7 critérios de presença obrigatória e 10 de presença complementar;

c) Deslocamento de Disco Crônico sem redução: contendo 4 critérios de presença obrigatória e 9 de presença complementar;

d) Deslocamento da ATM: contendo 2 critérios de presença obrigatória e 1 de presença complementar;

e) Sinovite/Capsulite: contendo 4 critérios de presença obrigatória e 10 de presença complementar;

f) Poliartrite: contendo 6 critérios de presença obrigatória e 10 de presença complementar;

g) Osteoartrite Primária: contendo 8 critérios de presença obrigatória e 4 de presença complementar;

h) Osteoartrite Secundária: contendo 7 critérios de presença obrigatória e 4 de presença complementar;

i) Anquilose Fibrosa: contendo 5 critérios de presença obrigatória;

j) Anquilose Óssea: contendo 6 critérios de presença obrigatória.

Para a avaliação do protótipo piloto, foram incluídas as 10 questões obrigatórias e as 6 de presença complementar referente ao deslocamento de disco com redução. O protótipo foi construído abrangendo os critérios de desenvolvimento de conteúdo (escolha do questionário de anamnese e seleção das questões), escolha do software de criação e formatação e layout.

Ao iniciar, o aplicativo mostrará a tela de "login". No primeiro acesso ao aplicativo, o usuário deverá realizar seu cadastro, nesse momento, será necessário o preenchimento de algumas informações, tais como: e-mail, nome, profissão, sexo, data de nascimento, cidade de residência e se possui algum conhecimento sobre DTM.

O usuário seguirá para as seguintes etapas:

1) ao entrar no aplicativo, será direcionado para a tela de login;

2) após o login, o usuário será direcionado para a tela de boas-vindas, contendo o logotipo do aplicativo;

3) seguirá para a tela onde responderá ao Índice Anamnésico de Fonseca e para detectar se há ou não presença de disfunção temporomandibular (DTM);

4) em caso positivo, surgirá uma próxima tela com ícones de todos os possíveis diagnósticos e será possível selecionar a realização de todos os testes ou somente alguns testes de diagnóstico;

5) ao fim da bateria de perguntas do módulo de diagnóstico selecionado, será exposta ao usuário a tela de resultados, na qual poderá conferir o percentual de possibilidade de determinado diagnóstico;

6) ao final, o usuário poderá salvar os resultados em seu dispositivo móvel, no formato pdf, que poderá ser enviado para seu e-mail e salvo no banco de dados.

Depois de validado através dos testes (estudo piloto), o aplicativo será indexado em lojas virtuais para aplicativos - Play Store - e na página da Universidade Luterana do Brasil, e ficará disponível para acesso dos universitários e interessados no tema.

RESULTADOS

As figuras a seguir apresentadas relacionam-se à funcionalidade do aplicativo móvel para diagnóstico das desordens temporomandibulares articulares. Após a tela de login (Figura 1), o usuário será direcionado para a tela de boas-vindas, de forma que, logo em seguida, inicia-se o questionário relativo ao Índice de Fonseca (Figura 2). As assertivas variam entre sim, não ou às vezes e, ao final, será emitido um valor correspondente ao grau de DTM que a pessoa é portadora. A presença deste índice visa facilitar a atuação do cirurgiãodentista na sua prática de atendimento. Caso o paciente em estudo possua DTM, independente do escore de severidade será dada sequência aos questionários relacionados ao diagnóstico de DTMs articular (Figura 3).

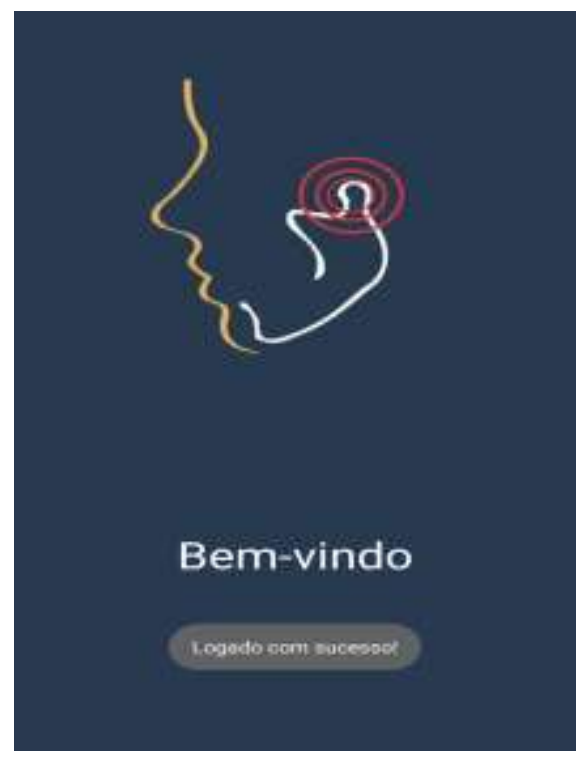

Figura 1:Tela de login e boas vindas. 


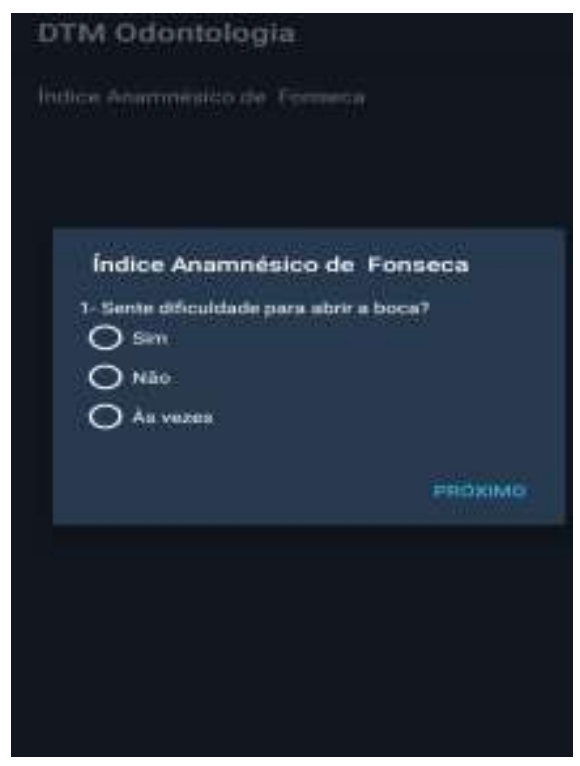

Figura 2: Início do índice anamnésico de Fonseca.

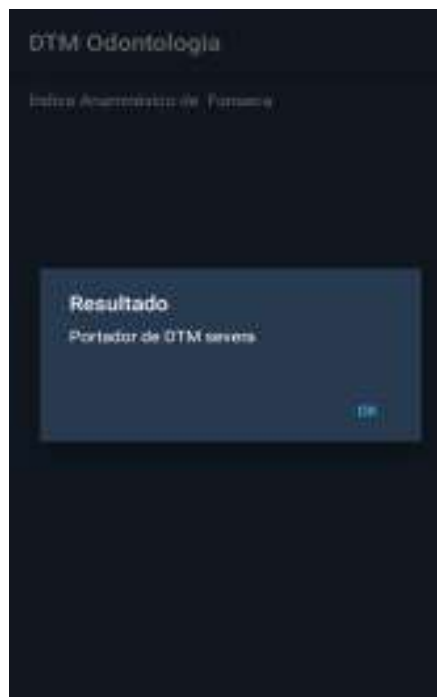

Figura 3: Exemplo de diagnóstico de DTM e sua severidade.

Neste primeiro momento do protótipo, foram introduzidas dez perguntas sobre 0 deslocamento do disco com redução (Figura 4), no qual o profissional irá responder com positivo se o paciente apresentar tal distúrbio (Figura 5).

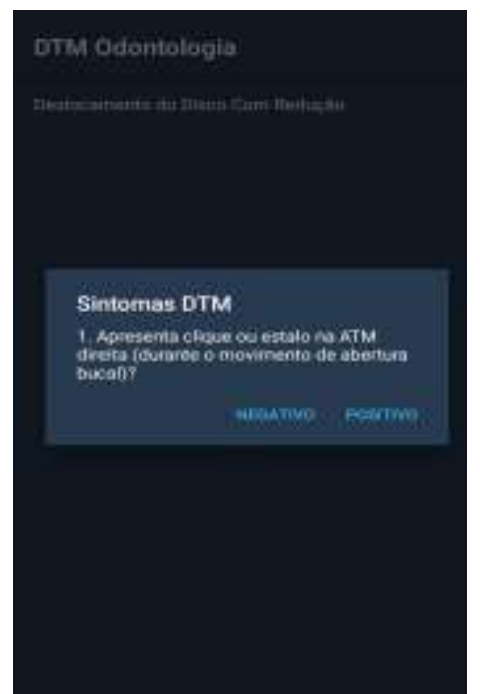

Figura 4: Exemplo de questão presente no aplicativo envolvendo DDCR.

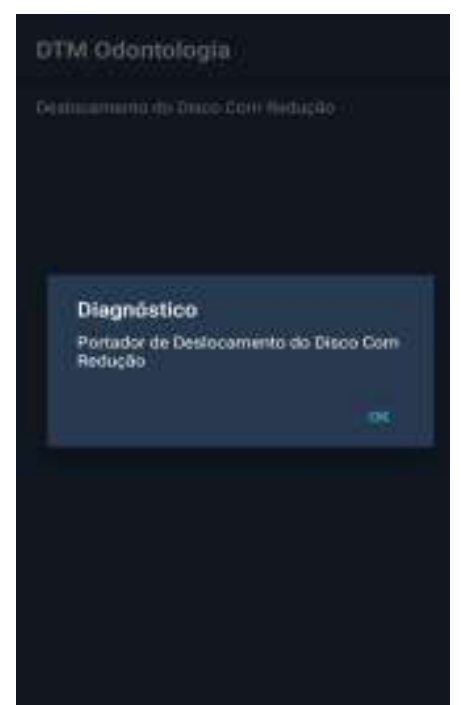

Figura 5: Diagnóstico final após responder o questionário

Caso uma destas perguntas tenha resposta negativa, o usuário será conduzido à página anterior sem um diagnóstico em relação ao deslocamento do disco com redução. Esta dinâmica procura garantir a agilidade processual e dinâmica ao fluxo de diagnóstico. É importante destacar, como viés do presente protótipo, a ausência de avaliação referente à portabilidade, confiabilidade e satisfação em relação ao aplicativo. De igual forma, serão acrescentadas as demais questões envolvendo outros distúrbios das DTMs articulares.

DISCUSSÃO

O sucesso de um tratamento odontológico integrado está relacionado com um diagnóstico preciso e o bom planejamento para estabelecer o plano de tratamento. A sistemática do exame clínico completo consiste em três etapas distintas, sendo elas a anamnese, o exame clínico e os exames complementares para a determinação do diagnóstico ${ }^{17}$. No contexto atual, a sociedade está utilizando, cada vez mais, em suas práticas laborais, as tecnologias de informação e comunicação disponíveis. Por seu caráter cada vez mais tecnológico, existe a demanda de gerar novos recursos que possam integrar e se aproximar do contexto dos profissionais ${ }^{18}$. Cabe destacar, o contexto favorável para o desenvolvimento de aplicativos móveis no âmbito da Odontologia. Em recente pesquisa, evidenciou-se que $81 \%$ dos cirurgiões-dentistas do Sistema Único de Saúde - SUS utilizam meios digitais para estudar e se atualizar ${ }^{19}$. Por exemplo, cita-se o desenvolvimento do aplicativo "PACOTAPS", para atenção primária em saúde (APS), com o objetivo de subsidiar gestores e equipes de saúde com informações sobre características da população de referência e da demanda atendida ${ }^{20}$. 
Em relação à ATM, pode-se encontrar um estudo no qual os autores criaram um aplicativo a fim de potencializar as habilidades na interpretação da articulação temporomandibular avaliada em imagens por Ressonância Magnética ${ }^{21}$. Tais estudos exemplificam a ampla variedade de aplicativos que estão em desenvolvimento ou já foram desenvolvidos. Dessa forma, a criação de um aplicativo que facilite o diagnóstico em relação às DTMs articulares torna-se muito importante. Além disso, até 0 presente momento, desconhece-se a existência de aplicativo móvel semelhante ao desenvolvido neste estudo, caracterizando a inovação do produto apresentado.

O aplicativo desenvolvido poderá contribuir positivamente no diagnóstico das DTMs articulares, pois sua elaboração está pautada em sólida base científica para auxiliar o profissional na tomada de decisão. Dessa forma, o referido aplicativo centralizou-se nas questões sobre DTMs Articulares, tendo em vista que as dores articulares acometem $62 \%$ dos pacientes ${ }^{22}$. Para o teste do protótipo, foram incluídos os 10 critérios obrigatórios para o diagnóstico de deslocamento de disco com redução descritos nos questionários. Estas questões foram escolhidas tendo em vista que os deslocamentos de disco com redução representam os estágios iniciais das desordens de desarranjo de disco ${ }^{23}$, além de apresentar prevalência estimada entre $20-35 \%$ da população, sendo muito mais prevalente que as demais patologias associadas ao disco articular $^{24}$.

É notório que a prática de utilização de aplicativos para diagnóstico ainda não está amplamente difundida, porém a maior barreira para uma ampla utilização acaba sendo uma questão cultural, na medida em que o cirurgiãodentista não está habituado a tal prática ${ }^{19}$. É relatado que o instrumento de triagem: Índice de Fonseca é um dos poucos instrumentos disponíveis em língua portuguesa que caracteriza e classifica a severidade dos sintomas de pacientes portadores de DTM ${ }^{25,26}$. Neste contexto, Adams ${ }^{27}$ (2016) relata que as pesquisas com aplicativos móveis dependem dos retornos e comentários dos usuários sobre quais recursos são considerados clinicamente relevantes. Assim, apenas com o uso do aplicativo poder-se-á avaliar sua acurácia diagnóstica em relação aos demais métodos.

Destaca-se que o aplicativo desenvolvido nesse estudo pode ser amplamente utilizado no ensino, sobretudo no âmbito da graduação em Odontologia, visto que não apresenta barreiras físicas nem custo elevado para sua implementação, mostrando-se uma boa alternativa para a educação continuada. Estudos conduzidos por Silva ${ }^{28}$ sugerem que estudantes do curso de Odontologia estão dispostos a experimentar novas formas de aprendizagem com 0 uso de ferramentas digitais, de forma que estratégias alternativas, tais como o aplicativo em questão, podem despertar o interesse dos alunos pelo estudo das patologias da ATM, capacitando-os, para prover assistência integral ao paciente.

CONCLUSÃO

A área da saúde tem experimentado novas formas de melhorar a prestação de serviço e ensino. O uso de aplicativos, por meio de dispositivos móveis, surge nesse contexto de modificações, facilitando a consulta a guidelines, ao diagnóstico e ao acompanhamento de pacientes. Destaca-se que esta plataforma digital não tem como propósito substituir qualquer exame complementar por imagem, mas sim apresentar uma forma complementar de auxílio diagnóstico para as patologias da ATM.

Entende-se que a criação de aplicativos móveis direcionados ao manejo da DTM é apropriada, já que a prevalência desta patologia é crescente em termos populacionais, sendo necessário que o cirurgião-dentista esteja capacitado para realizar o diagnóstico correto e propor estratégias de tratamento que garantam a qualidade de vida dos pacientes.

REFERÊNCIAS

1. Almeida AM, Fonseca F, Félix S. Dor orofacial e disfunções temporomandibulares: tratamento farmacológico. Portugal: Sociedade Portuguesa de Disfunção Temporomandibular e Dor Orofacial; 2016.

2. Teixeira SA, Almeida FM. A influência do tratamento ortodôntico nas disfunções temporomandibulares. Arq Bras Odontol. 2007; 2:129-36.

3. Figueiredo VMG, Cavalcanti $A L$, de Farias $A B$, Nascimento SR. Prevalência de sinais, sintomas e fatores associados em portadores de disfunção temporomandibular de disfunção temporomandibular. Acta Sci. Health Sci. 2009; 31(2):159-63.

4. Menezes MS, Bussadori SK, Fernandes KPS, Biasotto-Gonzalez DA. Correlação entre cefaleia e disfunção temporomandibulares. Fisioter pesq. 2008;15(2):183-87.

5. Tridapalli ALR, Salles BW. Tratamento fisioterápico das desordens temporomandibulares [monografia]. Florianópolis: Universidade Federal de Santa Catarina; 2012. 
6. Okeson JP. Tratamento das desordens temporomandibulares e oclusão. 7. ed. Rio de Janeiro: Elsevier; 2013.

7. Isidoro NV, Salles BW. Deslocamento do disco articular [monografia]. Florianópolis: Universidade Federal de Santa Catarina; 2005.

8. Mendes AG. Abortagem terapêutica da patologia inflamatória da articulação temporomandibular [dissertação]. Porto: Universidade Fernando Pessoa; 2012.

9. Santos PPA, Santos PRA, de Souza LB. Características gerais da disfunção temporomandibular: conceitos atuais. Rev Nav Odontol. 2009;3(1):10-3.

10. Francesquini LJ, Francesquini AM, Daruge E, Gonçalves RJ, Ambrosano GMB, Barbosa CMR et al. Disfunçäo de ATM: verificaçäo do conhecimento do cirurgiäo-dentista sobre etiologia, incidência e diagnóstico. J bras ortodon ortop facial. 1999; 4(19):67-79.

11. Moana EJF. Levantamento das atitudes e crenças dos ortodontistas com relação à disfunção têmporo-mandibular e dor orofacial. Rev Dent Press Ortodon Ortop Facial. 2005; 10(4):60-75.

12. Manfredi APS, Silva AA, Vendite LL. Avaliação da sensibilidade do questionário de triagem para dor orofacial e desordens temporomandibulares recomendado pela Academia Americana de Dor Orofacial. Rev Bras Otorrinolaringol. 2001; 67(6):763-68.

13. Google Painéis [homepage na internet]. Estatísticas de instalação do Google Play [acesso 10 mai 2018]. Disponível em: https:// developer.android.com/about/dashboards/

14. Faculdades de Informática de Taquara [homepage na internet]. SGBD MySQL [acesso em 12 mai 2018]. Disponível em: https://fit.faccat.br/ jonis/Artigo_mySQL.pdf

15. Google [homepage na internet]. Desenvolvedores Android [acesso em 12 mai 2018]. Disponível em: https://material.io/design/layout/understandinglayout.html

16. Fonseca DM, Bonfante G, Valle A, Freitas SFT. Diagnóstico pela anamnese da disfunção craniomandibular. Rev Gaúcha Odontol. 1994;42(1):23-8.

17. Rocha RG. Clínica integrada em odontologia. São Paulo: Artes Médicas; 2013.

18. Kohn $\mathrm{K}$, de Moraes $\mathrm{CH}$. O impacto das novas tecnologias na sociedade: conceitos e características da Sociedade da Informação e da Sociedade In: XXX Congresso Brasileiro de Ciências da Comunicação; 2007, 29 de agosto a 2 de setembro; Santos (SP). Intercom Sociedade Brasileira de Estudos Interdisciplinares da Comunicação.

19. Lemos SGD, Maria MC, Carloto EET. Recursos utilizados por graduandos de odontologia e cirurgiões-dentistas do SUS para estudar e resolver dúvidas surgidas durante o trabalho. Rev Abeno. 2014;14(1):3-16.

20. Tomasi E, Facchini LA, Osorio A, Fassa AG. Aplicativo para sistematizar informações no planejamento de ações de saúde pública. Rev Saúde Pública. 2003;37(6):800-806.

21. Arús NA. Desenvolvimento e teste de um objeto digital de aprendizagem para interpretação das imagens por ressonância magnética da articulação temporomandibular [tese]. Porto Alegre: Universidade Federal do Rio Grande do Sul; 2012.

22. De Siqueira JTT, Teixeira MJ. Dores orofaciais: diagnóstico e tratamento. São Paulo: Artes Médicas; 2012.

23. Dantas AMX, dos Santos EJL Vilela RM, de Lucena LBS. Perfil epidemiológico de pacientes atendidos em um Serviço de Controle da Dor Orofacial. Rev Odontol UNESP. 2015; 44(6):313-19.

24. Maydana AV, Tesch RDD, Denardin OVP, Ursi WJdS, Dworkin SF. Possíveis fatores etiológicos para desordens temporomandibulares de origem articular com implicações para diagnóstico e tratamento. Dental Press J. Orthod. 2010;15(3):78-86.

25. Chaves TC, de Oliveira AS, Grossi DB, Chaves TC. Main instruments for assessing temporomandibular disorders, part I: indices and questionnaires; a contribution to clinicians and researchers. Fisioter Pesq. 2008;15(1): 92-100.

26. Pedrosa AS. Propriedades de medida do índice Anamnésico de Fonseca. São Paulo [dissertação]. São Paulo: Faculdade de Fisioterapia da Universidade de São Paulo; 2011.

27. Adams BW. Automation and apps for clinical dental biomechanics. Cranio. 2016;34(5): 343-47.

28. Silva LA. Desenvolvimento de aplicativo como objeto de aprendizagem: atlas virtual e interativo para ensino da anatomia cabeça e pescoço direcionado à Odontopediatria. Dissertação. Bauru: Faculdade de Odontologia de Bauru da Universidade de São Paulo; 2016. 


\section{CONFLITO DE INTERESSES}

Os autores declaram não haver conflitos de interesse

\section{AUTOR PARA CORRESPONDÊNCIA}

\section{Caren Serra Bavaresco}

Curso de Graduação em Odontologia

ULBRA Univ. Luterana do Brasil

92425-020 Canoas - RS, Brasil

E-mail: c_bavaresco@yahoo.com.br

Submetido em 11/05/2020

Aceito em 23/10/2020 